Original Research Paper

\title{
Involvement of the $\alpha / \beta$ Isoform of p38 MAP Kinase in Chemotactic Responses of Human Eosinophils to Eotaxin (CCL11) and RANTES (CCL5)
}

\author{
Anwar M Hasan and Gordon Dent \\ Institute of Science and Technology in Medicine, \\ Faculty of Medicine and Health Sciences, Keele University, Keele, Staffordshire ST5 5BG, UK
}

Article history

Received: 07-06-2017

Revised: 17-06-2017

Accepted: 19-10-2017

Corresponding Author: Gordon Dent

Institute of Science and Technology in Medicine, Faculty of Medicine and Health Sciences, Keele University, Keele, Staffordshire ST5 5BG, UK

Tel +4401782 734652

Email: g.dent@keele.ac.uk

\begin{abstract}
Eosinophils are the principal effector cells for allergic inflammation in a variety of diseases, in which they contribute to tissue damage and remodelling processes via the secretion of cytotoxic granular proteins and cytokines. The intracellular mechanisms that control the activation, recruitment and survival of eosinophils are fundamental in understanding these disease processes. Phosphoinositide 3-kinase (PI3K) has been shown previously to be essential for eosinophil chemotactic responses to some stimuli but not others. Human blood neutrophils have been shown to utilize two antagonistic signalling pathways for chemotaxis: PI3K and p38 mitogen-activated protein kinase (p38 MAPK). In the present study, the role of p38 MAPK in chemotactic responses of an eosinophildifferentiated myeloid leukaemia cell line (EOL-1) and human peripheral blood eosinophils to a range of stimuli - platelet-activating factor (PAF), eotaxin 1 (CCL11), RANTES (CCL5), interleukin 8 (IL8, CXCL8) and IL16 - was explored through the use of the p38 MAPK $\alpha / \beta$ isoform inhibitor, SB 203580. SB 203580 caused significant inhibition of chemotactic responses of both EOL-1 cells and blood eosinophils to eotaxin 1 and RANTES ( $\geq 75 \%$ inhibition at $1 \mu \mathrm{M}$ SB $203580, p<0.01$ ) but had no effect on the migration induced by PAF and IL16 $(<25 \%)$ and little or no effect on responses to IL8. Responses to PAF - but not eotaxin - have been shown previously to be suppressed by PI3K inhibition. The complementary pattern of inhibition observed in the present study provides evidence that distinct PI3K-dependent and p38 MAPK-dependent chemoattractants may also exist for eosinophils.
\end{abstract}

Keywords: Eosinophils, Chemotaxis, Cell Signalling, p38 MAP Kinase

\section{Introduction}

Eosinophils are initiator and effector cells of Th2mediated immunity (Ravin and Loy, 2016). They are associated with key components of inflammation in a variety of chronic diseases affecting the airways (Eng and DeFelice, 2016), heart (Séguéla et al., 2015) and gastrointestinal system (Hogan et al., 2013). Eosinophils are characterized by secretory granules that contain multiple cytotoxic proteins (Ravin and Loy, 2016). Additionally, eosinophils produce reactive oxygen metabolites, lipid mediators and cytokines, all of which are capable of causing severe host tissue damage in eosinophilic inflammation (Barnes, 2011).
Eosinophil migration appears to be modulated by two fundamental processes: Cell adhesion systems located at the site of inflammation in local endothelium and epithelium and chemotactic signals elicited through cytokine, chemokine and other chemoattractant receptors (Lampinen et al., 2004). A range of inflammatory mediators and cytokines have been identified as eosinophil attractants and activators, including plateletactivating factor, $\mathrm{CC}$ chemokines including eotaxin (CCL11) (Mishra et al., 2005) and regulated on activation normal T-cell expressed and secreted (RANTES, CCL5) (Svensson et al., 2009), CXC chemokines including interleukin 8 (IL8, CXCL8) (Bates et al., 2010) and the lymphocyte chemoattractant factor interleukin 16 (IL16) 
(Rand et al., 1991). The cellular signalling pathways responsible for evoking and regulating eosinophil chemotaxis toward these chemoattractants are incompletely understood, although a role for receptormediated activation of phosphoinositide 3-kinase (PI3K) in responses to certain stimuli has been identified (Mishra et al., 2005).

The p38 MAP kinase (MAPK) signalling pathway plays an important role in inflammation and other physiological processes. Specific inhibitors of p38 MAPK $\alpha$ and $\beta$ block production of the major inflammatory cytokines (e.g. tumour necrosis factor $\alpha$ and interleukin 1) and other proteins (e.g. cyclooxygenase 2) and are anti-inflammatory in animal models of disease (Ono and Han, 2000). A major function of the pathway is post-transcriptional control of inflammatory gene expression. Inhibitors of p38 MAPK have been shown to reduce inflammatory cytokine production and eosinophil infiltration into the lungs in animal models of asthma (Underwood et al., 2000) and to inhibit angiogenesis in a murine model of rheumatoid arthritis (Jackson et al., 1998).

SB203580 is a selective inhibitor of the $\alpha$ and $\beta$ isoforms of p38 MAPK that inhibits the cytokineinduced adhesion, shape change and transmigration of eosinophils (Ip et al., 2003), as well as inhibiting eotaxin 1 production in human primary lung fibroblasts (Rokudai et al., 2006) and capsaicin-induced production of PAF in oesophageal epithelial cells (Ma et al., 2010). Pharmacological inhibition of receptors and signal transduction molecules in human eosinophils has shown that the release of oxygen free radicals in response to eotaxin 1 is relatively more dependent on the p38 MAPK pathway than the response to bacterial formyl peptide chemoattractants (Svensson et al., 2009).

Studies in human neutrophils revealed a signalling hierarchy for chemoattractants in which responses to specific attractants exhibited a dependence upon either PI3K or p38 MAPK (Heit et al., 2007). Since we have shown previously that eosinophil responses to certain attractants are dependent upon PI3K while others are not (Mishra et al., 2005; Hasan et al., 2010), we undertook a study to determine whether eosinophil chemotactic responses to a number of factors-including those known to be PI3K-independent-involved p38 MAPK.

\section{Materials and Methods}

\section{Cells}

The human leukaemia cell line, EoL-1 was cultured and differentiated to an eosinophilic phenotype as described previously (Al-Rabia et al., 2004). Cell viability was determined every 2 days by trypan blue dye exclusion. Viability was maintained at $>90 \%$ throughout cultures.
Human peripheral blood eosinophils were isolated from non-asthmatic volunteers as described previously (Dent et al., 1998). Blood donors gave informed consent to the use of their cells and the project was approved by the North Staffordshire Local Research Ethics Committee (reference 06/Q2604/15).

\section{Chemotaxis Assay}

Chemotactic responses of differentiated EoL-1 cells and human peripheral blood eosinophils were measured in a 96-well blind chamber assay using $5 \mu \mathrm{m}$ pore-size filters, as described previously (Mishra et al., 2005).

\section{Statistical Analysis}

Data are expressed as arithmetic mean \pm standard error of the mean (SEM) from the indicated numbers of experiments. Statistical analyses were performed using SPSS version 21 for PC (IBM Corp., Armonk NY, USA). For comparisons of multiple groups (i.e. varying concentrations of SB 203580), repeatedmeasures analysis of variance (ANOVA) was followed by post hoc pairwise comparisons with untreated (control) cells using Dunnett's test for multiple comparisons. A probability $(P)<0.05$ was defined as significant throughout.

\section{Results}

Effect of $\alpha / \beta$ p38 MAPK Inhibitor (SB 203580) on Chemotactic Responses of EOL-1 Cells and Human Peripheral Blood Eosinophils to Eotaxin 1 and $P A F$

Eosinophil-differentiated EoL-1 cells exhibited chemotactic responses to both PAF and eotaxin 1, with optimal concentrations of $100 \mathrm{nM}$ and $30 \mathrm{nM}$, respectively (Dent et al., 1998). Chemotactic responses to eotaxin 1 were inhibited in a concentrationdependent manner by SB 203580. In contrast, inhibition of PAF-induced chemotactic responses by SB 203580 did not achieve statistical significance at concentrations up to $10 \mu \mathrm{M}$ (Fig. 1A).

Both PAF and eotaxin 1 stimulated migration of human peripheral blood eosinophils, with optimal concentrations of $30 \mathrm{nM}$ for each stimulus (Dent et al., 1998). The response to eotaxin was inhibited concentration-dependently by SB 203580, while the drug had no significant effect on the response to PAF at concentrations of $100 \mu \mathrm{M}$ (Fig. 1B).

\section{Effect of SB 203580 on Chemotactic Responses of} EOL-1 Cells to IL8, IL16 and RANTES

In preliminary experiments, the optimal concentration of IL8 for induction of both 
differentiated EOL-1 cell and blood eosinophil chemotaxis was found to be $10 \mathrm{nM}$, while that for RANTES and IL16 was 0.1 nM (Hasan, 2008). Chemotactic responses to RANTES were inhibited by SB 203580 in a concentration-dependent manner (Fig. 2A). SB 203580 had no significant effect on chemotactic responses to IL16 or IL8 at concentrations up to $10 \mu \mathrm{M}$ (Fig. $2 \mathrm{~B}$ and $2 \mathrm{C}$ ).
Effect of SB 203580 on Chemotactic Responses of Human Peripheral Blood Eosinophils to IL8, IL16 and RANTES

SB203580 inhibited the chemotactic response of human peripheral blood eosinophils to RANTES in a concentration-dependent manner (Fig 3A). SB 203580 had no significant effect on chemotactic responses to IL16 or IL8 at concentrations up to $10 \mu \mathrm{M}$ (Fig 3B \& C).
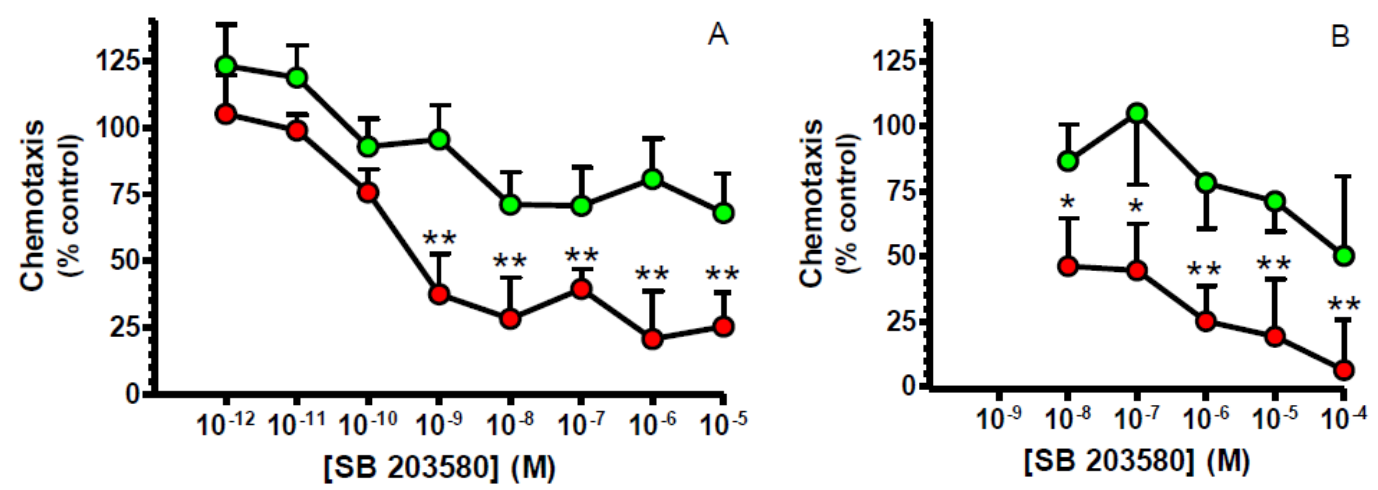

Fig. 1. Effects of SB 203580 on (A) eosinophil cell line EoL-1 and (B) human peripheral blood eosinophil chemotactic responses to $100 \mathrm{nM}$ PAF $(\bullet)$ and $30 \mathrm{nM}$ eotaxin $1(\bullet)$. Data are shown as mean \pm SEM from six experiments conducted in triplicate for each inhibitor combination. ${ }^{*} p<0.05,{ }^{*} \mathrm{p}<0.01$ compared with control response in the absence of inhibitor by repeatedmeasures ANOVA and post hoc Dunnett's test.
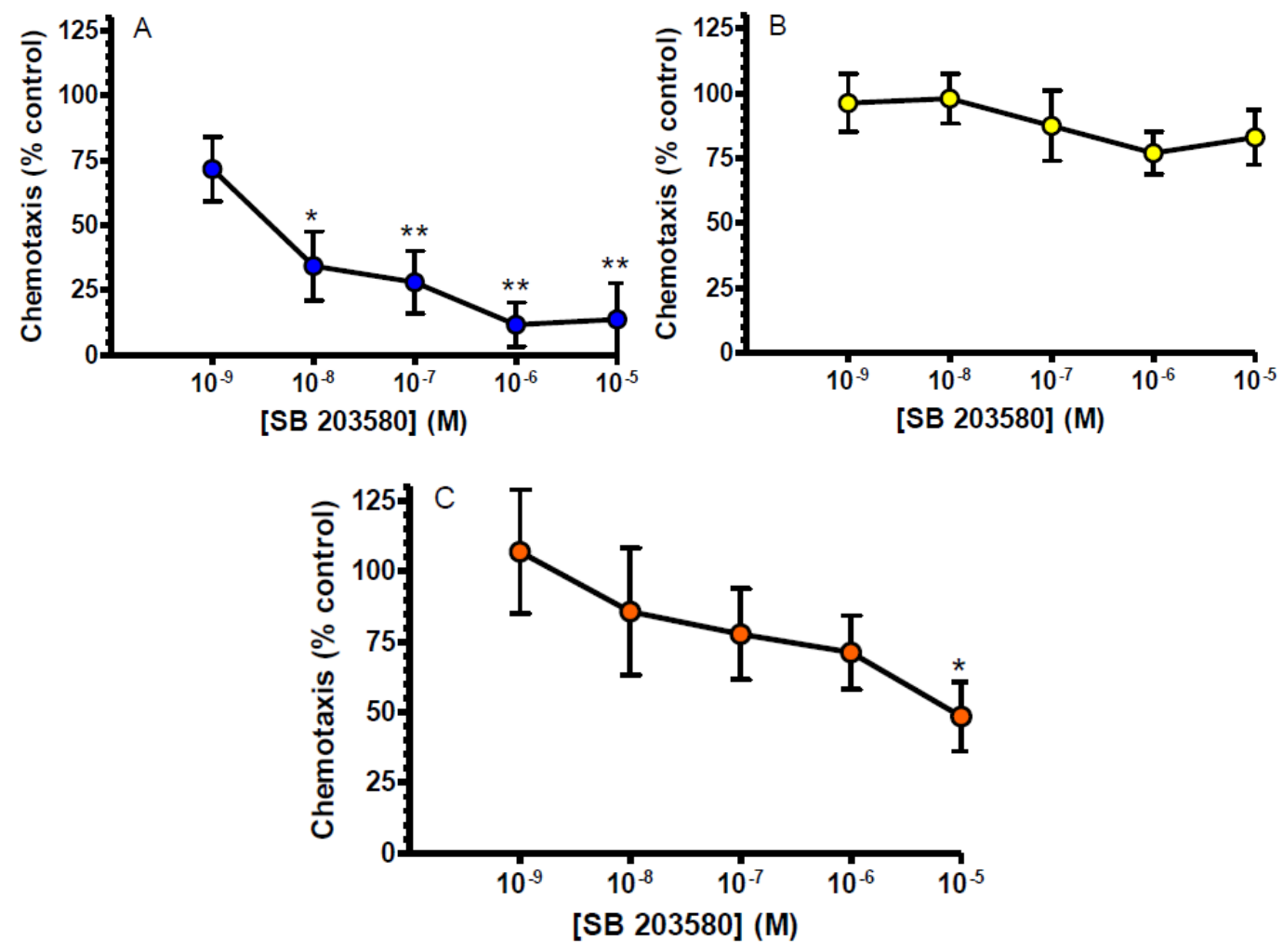

Fig. 2. Effects of SB 203580 on EoL-1 cell chemotactic responses to (A) $0.1 \mathrm{nM}$ RANTES, (B) $0.1 \mathrm{nM}$ IL16 and (C) $10 \mathrm{nM}$ IL8. Data are shown as mean \pm SEM from four experiments conducted in triplicate for each inhibitor combination. ${ }^{*} p<0.05$, $*^{*} p<0.01$ compared with control response in the absence of inhibitor 

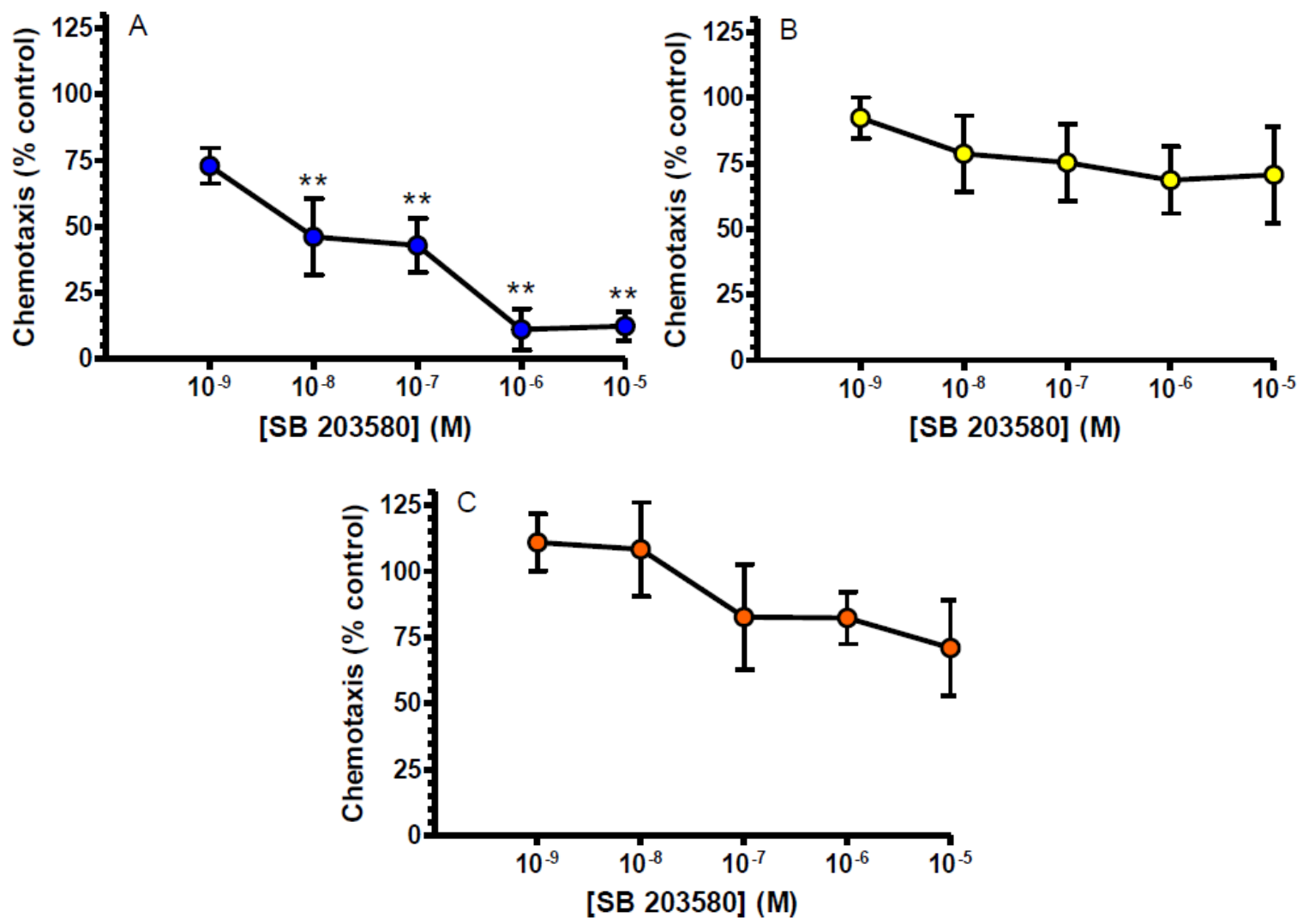

Fig. 3. Effects of SB 203580 on blood eosinophil chemotactic responses to (A) $0.1 \mathrm{nM}$ RANTES, (B) $0.1 \mathrm{nM}$ IL16 and (C) $10 \mathrm{nM}$ IL8. Data are shown as mean \pm SEM from four experiments conducted in triplicate for each inhibitor combination. ${ }^{* * p} p<0.01$ compared with control response in the absence of inhibitor

\section{Discussion}

Previous studies have shown a differential dependence of chemotactic responses in human eosinophils and eosinophil-differentiated EOL-1 cells upon PI3K. Responses to the phospholipid inflammatory mediator platelet-activating factor (PAF) are highly sensitive to suppression by PI3K inhibitors (Mishra et al., 2005), with the PI3K $\gamma$ isoform specifically essential to these responses (Hasan et al., 2010). In contrast, responses to the $C C$ chemokine eotaxin 1 - a key eosinophil chemoattractant in airway secretions in severe asthma (Dent et al., 2004) - are unaffected by PI3K inhibitors (Mishra et al., 2005; Hasan et al., 2010). It is, therefore, apparent that chemotactic responses of eosinophils are not mediated by a single intracellular signalling pathway.

In a landmark study Heit et al. (2002) demonstrated the existence of a signalling hierarchy that allows neutrophils to migrate towards higher concentrations of specific chemoattractants against concentration gradients of other attractants. Heit et al. (2002) classified these two types of substance as intermediary chemoattractants (e.g. IL8 and leukotriene $\mathrm{B}_{4}$ ), with actions mediated by PI3K and end-stage chemoattractants (e.g. complement anaphylotoxin $\mathrm{C} 5 \mathrm{a}$ and bacterial formyl peptide fMetLeu-Phe) whose actions are mediated by p 38 MAPK.

In the present study, chemotactic responses of eosinophils to a range of stimuli were assessed to determine their sensitivity to an inhibitor of the $\alpha / \beta$ isoforms of $\mathrm{p} 38$ MAPK, SB 203580.

In a complementary finding to that of our previous reports (Mishra et al., 2005; Hasan et al., 2010), we found that responses to eotaxin 1 were highly sensitive to inhibition by SB 203580 while those to PAF were not. Among the other stimuli studied, another CC chemokine - RANTES - also exhibited chemotactic responses sensitive to p38 MAPK inhibition. While both of these chemokines are able to exert actions through multiple receptors, they have a single common target expressed in eosinophils: the CCR3 receptor (Elsner et al., 2004). This may account for the shared dependence of responses to these two stimuli upon the same signalling pathway.

Eotaxin may stimulate chemotaxis through activation of one or more protein kinases, which then activate effector molecules that recruit p38 MAPK. These in turn activate downstream molecules which induce 
polarization and cytoskeletal reorganization. Thus, eotaxin and RANTES may induce the chemotaxis of eosinophils by a p38 MAPK-dependent mechanism. The results of the present study are similar to those reported by Stubbs et al in 2002, which showed evidence of involvement of p38 MAPK in multiple eosinophil responses to eotaxin (Stubbs et al., 2002).

While the CXC chemokine IL8 is recognized as having chemoattractant activity for eosinophils, the signalling pathways involved in this response are poorly understood. IL8 activates MAP kinases 3 and 1 (ERK1 and ERK2) in IL5-primed eosinophils and this action is necessary for the induction of leukotriene $\mathrm{C}_{4}$ synthesis (Bates et al., 2000), however the signal transduction events involved in chemotaxis have not been identified. Although IL8 was classified as an intermediate (PI3Kdependent) attractant for neutrophils (Heit et al., 2002), the receptor presumed to mediate this response in neutrophils - CXCR1 - has not been shown to be expressed by eosinophils (Liu et al., 2003). We demonstrate here that IL8-induced eosinophil chemotaxis does not exhibit the same degree of dependence upon p38 MAPK as the CCR3 agonists eotaxin 1 and RANTES, with only marginal levels of inhibition (up to approximately $25 \%$ ) observed in the presence of SB 203580 at concentrations that produce approximately $75 \%$ inhibition of responses to the $\mathrm{CC}$ chemokines. It remains to be determined which other signalling pathways-possibly including PI3K-dependent mechanisms-contribute to CXC chemokine chemotaxis responses in eosinophils.

The lymphocyte chemoattractant factor, IL16, induces activation and suppressor events in $T$ lymphocytes through a variety of mechanisms involving multiple receptors (Lynch et al., 2003). While CD4 is the key receptor for IL16 actions on T lymphocytes, nonlymphoid immune effector cells may utilize other receptors for this cytokine and a role for PI3K in chemotactic responses to IL16 in mast cells has been demonstrated (Qi et al., 2006). In the present study there was no apparent dependence upon p38 MAPK of eosinophil chemotactic responses to IL16, with no inhibition of responses observed at any concentration of SB 203580. While this cannot be taken as evidence of involvement of another specific signalling pathway, it does contribute to an overall picture of stimuli shown elsewhere to evoke PI3K-dependent responses exhibiting no dependence upon p38 MAPK in our studies.

Future research studies should focus on stimulus, response and cell-type heterogeneity of p38 MAPK dependence. The present study has utilized a single inhibitor of the $\alpha / \beta$ isoforms of the enzyme. Further studies are required to investigate the functions of the individual isoforms $\alpha, \beta, \delta$ and $\gamma$ of p38 MAPK and the mechanisms by which they contribute to activation of human eosinophils. The interference or cross-talk of this pathway (p38 MAPK pathway) with the other pathways that influence the migration and accumulation of human eosinophils will be an interesting field for future studies.

\section{Conclusion}

Chemotactic responses of human eosinophils to $\mathrm{CC}$ chemokines (eotaxin 1 and RANTES) are dependent upon $\alpha / \beta$ p38 MAPK. Responses to PAF and IL16 are not dependent on $\alpha / \beta$ p38 MAPK, while responses to a CXC chemokine (IL8) show partial dependence. The dependence of responses of PAF and eotaxin 1 on $\alpha / \beta$ p38 MAPK are a mirror image of the dependence of these responses on PI3K, suggesting that different classes of chemoattractants utilizing different intracellular signaling pathways may exist for eosinophils, as has been described for human neutrophils.

\section{Acknowledgment}

The authors wish to thank Prof Garry Walsh (University of Aberdeen) for advice on the culturing and differentiation of EOL-1 cells.

\section{Author's Contributions}

Anwar M Hasan: Conducted the laboratory work and the background literature review, summarized the data and drafted the manuscript.

Gordon Dent: Formulated the research question, designed the experimental protocol, conducted statistical analyses, produced the final graphs and edited the manuscript for submission.

\section{Ethics}

This work does not present any ethical issues.

\section{References}

Al-Rabia, M.W., M.G. Blaylock, D.W. Sexton and G.M. Walsh, 2004. Membrane receptor-mediated apoptosis and caspase activation in the differentiated EoL-1 eosinophilic cell line. J. Leukoc Biol., 75: 1045-1055. DOI: 10.1189/jlb.0803404

Barnes, P.J., 2011. Pathophysiology of allergic inflammation. Immunol. Rev., 242: 31-50. DOI: 10.1111/j.1600-065X.2011.01020.X

Bates, M.E., J.B. Sedgwick, Y. Zhu, L.Y. Liu, R.G. Heuser et al., 2010. Human airway eosinophils respond to chemoattractants with greater eosinophilderived neurotoxin release, adherence to fibronectin and activation of the Ras-ERK pathway when compared with blood eosinophils. J. Immunol., 184: 7125-7133. DOI: 10.4049/jimmunol.0900634 
Bates, M.E., V.L. Green and P.J. Bertics, 2000. ERK1 and ERK2 activation by chemotactic factors in human eosinophils is interleukin 5-dependent and contributes to leukotriene $\mathrm{C} 4$ biosynthesis. J. Biol. Chem., 275: 10968-10975.

DOI: $10.1074 /$ jbc.275.15.10968

Dent, G., C. Hadjicharalambous, T. Yoshikawa, R.L. Handy, J. Powell et al., 2004. Contribution of eotaxin-1 to eosinophil chemotactic activity of moderate and severe asthmatic sputum. Am. J. Respir. Crit. Care Med., 169: 1110-1117.

DOI: $10.1164 / \mathrm{rccm} .200306-8550 \mathrm{C}$

Dent, G., N.M. Muñoz, E. Rühlmann, X. Zhu, A.R. Leff et al., 1998. Protein kinase C inhibition enhances platelet-activating factor-induced eicosanoid production in human eosinophils. Am. J. Respir. Cell Mol. Biol., 18: 136-144.

DOI: 10.1165/ajrcmb.18.1.2817

Elsner, J., S.E. Escher and U. Forssmann, 2004. Chemokine receptor antagonists: A novel therapeutic approach in allergic diseases. Allergy, 59: $1243-1258$.

DOI: 10.1111/j.1398-9995.2004.00710.x

Eng, S.S. and M.L. DeFelice, 2016. The role and immunobiology of eosinophils in the respiratory system: A comprehensive review. Clin. Rev. Allergy Immunol., 50: 140-158. DOI: $10.1007 / \mathrm{s} 12016-015-8526-3$

Hasan, A.M., 2008. Intracellular signalling in eosinophils: Differential roles for phosphoinositide 3-kinase and p38 MAP kinase in cellular responses to chemoattractants. PhD Thesis, Keele University.

Hasan, A.M., M. Mourtada-Maarabouni, M.S. Hameed, G.T. Williams and G. Dent, 2010. Phosphoinositide 3-kinase gamma mediates chemotactic responses of human eosinophils to platelet-activating factor. Int. Immunopharmacol., 10: 1017-1021.

DOI: 10.1016/j.intimp.2010.05.014

Heit, B., S. Tavener, E. Raharjo and P. Kubes, 2002. An intracellular signaling hierarchy determines direction of migration in opposing chemotactic gradients. J. Cell Biol., 159: 91-102. DOI: 10.1083/jcb.200202114

Hogan, S.P., A. Waddell and P.C. Fulkerson, 2013. Eosinophils in infection and intestinal immunity. Curr. Opin. Gastroenterol., 29: 7-17. DOI: 10.1097/MOG.0b013e32835ab29a

Ip, W.K., C.K. Wong, C.B. Wang, Y.P. Tian and C.W. Lam, 2003. Interleukin-3,-5 and granulocyte macrophage colony-stimulating factor induce adhesion and chemotaxis of human eosinophils via p38 mitogen-activated protein kinase and nuclear factor $\kappa$ B. Immunopharmacol. Immunotoxicol., 27: 371-393. DOI: 10.1165/rcmb.2002-0289OC
Jackson, J.R., B. Bolognese, L. Hillegrass, S. Kassis, J. Adams et al., 1998. Pharmacological effects of SB 220025, a selective inhibitor of p38 mitogenactivated protein kinase, in angiogenesis and chronic inflammatory disease. J. Pharmacol. Exp. Ther., 284: 687-692.

Lampinen, M., M. Carlson, L.D. Hakansson and P. Venge, 2004. Cytokine-regulated accumulation of eosinophils in inflammatory disease, Allergy, 59: 793-805. DOI: 10.1111/j.1398-9995.2004.00469.x

Liu, L.Y., N.N. Jarjour, W.W. Busse and E.A. Kelly, 2003. Chemokine receptor expression on human eosinophils from peripheral blood and bronchoalveolar lavage fluid after segmental antigen challenge. J. Allergy Clin. Immunol., 112: 556-562.

Lynch, E.A., C.A. Heijens, N.F. Horst, D.M. Center and W.W. Cruikshank, 2003. IL-16/CD4 preferentially induces Th1 cell migration: Role of CCR5. J. Immunol., 171: 4965-4968. DOI: $10.4049 /$ jimmunol.171.10.4965

Ma, J., K.M. Hamett, J. Behar, P. Biancani and W. Cao, 2010. Signaling in TRPV1-induced platelet activating factor (PAF) in human esophageal epithelial cells. Am. J. Physiol. Gastrointest. Liver Physiol., 298: G233-G240.

DOI: 10.1152/ajpgi.00409.2009

Mishra, R.K., J.E. Scaife, Z. Harb, B.C. Gray, R. Djukanovic et al., 2005. Differential dependence of eosinophil chemotactic responses on phosphoinositide 3-kinase (PI3K). Allergy, 60: 1204-1207.

DOI: $10.1111 / \mathrm{j} .1398-9995.2005 .00845 . \mathrm{x}$

Ono, K. and J. Han, 2000. The p38 signal transduction pathway: activation and function. Cell Signal., 12: 1-13. DOI: 10.1016/S0898-6568(99)00071-6

Qi, J.C., J. Wang, S. Mandadi, K. Tanaka, B.D. Roufogalis et al., 2006. Human and mouse mast cells use the tetraspanin CD9 as an alternate interleukin-16 receptor. Blood, 107: 135-142. DOI: $10.1182 /$ blood-2005-03-1312

Rand, T.H., W.W. Cruikshank, D.M. Center and P.F. Weller, 1991. CD4-mediated stimulation of human eosinophils: lymphocyte chemoattractant factor and other CD4-binding ligands elicit eosinophil migration. J. Exp. Med., 173: 1521-1528. DOI: $10.1084 /$ jem.173.6.1521

Ravin, K.A. and M. Loy, 2016. The eosinophil in infection. Clin. Rev. Allergy Immunol., 50: 214227. DOI: $10.1007 / \mathrm{s} 12016-015-8525-4$

Rokudai, A., Y. Terui, R. Kuniyoshi, Y. Mishima and Y. Mishima et al., 2006. Differential regulation of eotaxin-1/CCL11 and eotaxin-3/CCL26 production by the TNF- $\alpha$ and IL-4 stimulated human lung fibroblast. Biol. Pharm. Bull., 29: 1102-1109. DOI: $10.1248 / \mathrm{bpb} .29 .1102$ 
Séguéla, P.E., X. Iriart, P. Acar, M. Montaudon and R. Roudaut et al., 2015. Eosinophilic cardiac disease: Molecular, clinical and imaging aspects. Arch. Cardiovasc Dis., 108: 258-268.

DOI: $10.1016 /$ j.acvd.2015.01.006

Stubbs, V.E.L., P. Schrahtl, A. Hartnell, T.J. Williams, B.A. Peskar et al., 2002. Indomethacin causes prostaglandin D2-like and eotaxin-like selective responses in eosinophils and basophils. J. Biol. Chem., 277: 26012-26020.
Svensson, L., E. Redvall, M. Johnsson, A.L. Stenfeldt, C. Dahlgren et al., 2009. Interplay between signaling via the Formyl Peptide Receptor (FPR) and Chemokine Receptor 3 (CCR3) in human eosinophils. J. Leukoc Biol., 86: 327-336. DOI: 10.1189/jlb.0908514

Underwood, D.C., R.R. Osborn, S. Bochnowicz, E.F. Webb, D.J. Rieman et al., 2000. SB 239063, a p38 MAPK inhibitor, reduces neutrophilia, inflammatory cytokines, MMP-9 and fibrosis in lung. Am. J. Physiol. Lung Cell Mol. Physiol., 279: L895-L902. 